TRENDS IN HYDROZOAN BIOLOGY - IV. C.E. MILLS, F. BOERO, A. MIGOTTO and J.M. GILI (eds.)

\title{
Kinematic comparison of bell contraction by four species of hydromedusae*
}

\author{
M.D. FORD ${ }^{1,3}$ and J.H. COSTELLO ${ }^{2}$ \\ ${ }^{1}$ Department of Marine Sciences, University of Connecticut, 1084 Shennecossett Road, Groton, CT 06340 \\ ${ }^{2}$ Corresponding Author: Biology Department, Providence College, Providence, RI 02819 \\ ${ }^{3}$ Present address: NOAA/NESDIS/NODC, SSMC3, 4th floor, OCI 1315 East West Highway, \\ Silver Spring, MD 20910 USA
}

\begin{abstract}
SUMMARY: Bell form strongly affects the hydrodynamic performance of swimming hydromedusae. Although the relationship between bell shape and hydrodynamic parameters has been documented for static models of different bell shapes, the dynamic differences in contraction characteristics of different bell shapes have not been described. This is an important issue in medusan motion because the way in which medusan bells contract may influence the effect of bell shape on swimming performance. We measured differences in bell morphologies and wake velocities during swimming by two prolate (streamlined) and two oblate (disc shaped) forms of hydromedusae. Our results indicate that propulsion by prolate medusae is fundamentally different than that of oblate forms. Prolate species were characterized by contraction over the entire length of the bell and produced a narrower, higher velocity jet. Oblate medusae contracted primarily near the bell margin, and produced a broader, lower velocity jet. Prolate medusae achieved higher velocities but lower Froude propulsion efficiencies than oblate medusae. The adaptive value of these patterns are interpreted in terms of foraging and life history patterns.
\end{abstract}

Key words: swiming, morphology, efficience, fluid flow, jet propulsion, foraging.

\section{INTRODUCTION}

The great variety of bell morphologies among the hydromedusae (Russell 1953, Kramp 1959) have clear hydrodynamic correlates (Daniel 1983, 1984, 1985; Colin and Costello 1996) which are related to foraging mode (Costello 1992). Medusae that sit in ambush for prey do not typically swim while capturing prey. For these species, swimming serves to change position of the medusa, either for escape or migration to a new feeding location. Rapid acceleration is crucial for escape swimming and prolate bell forms are optimally designed for

\footnotetext{
*Received February 20, 1999. Accepted June 26, 1999
}

rapid accelerations (Daniel 1983, Colin and Costello 1996). As a result, bell form among ambush-foraging medusae may be expected to fall towards the prolate end of the spectrum among hydromedusan bell shapes. In contrast, hydromedusae that entrain and capture prey by utilizing the currents created during swimming are more suited to oblate bell shapes. The reason for this is that an oblate form, although minimizing acceleration of the medusa's body, maximizes the acceleration of fluid surrounding the medusa's bell (Daniel 1983, 1985; Colin and Costello 1996). It is this fluid, known as the added-mass of the medusa's bell, that contains the prey that are sieved through the tentacles hanging in the wake created by the medusa. By maxi- 
mizing the volume of their wake, oblate medusae maximize the volume of fluid filtered for prey (Colin and Costello, 1996).

Although there is clear evidence that static models of different shapes affect hydrodynamic variables such as drag and the acceleration reaction (Daniel, 1985), the possibility that bell forms have different dynamic traits has remained unexplored. Specifically, we wanted to address the possibility that medusae of different bell forms contracted their bells differently. In order to resolve this question, we examined two representative prolate [Aglantha digitale (O.F. Muller, 1776), Sarsia sp. (probably Sarsia apicula (Murbach and Shearer, 1902), but also frequently referred to as Sarsia tubulosa in the local literature (A. BrinckmannVoss, pers. comm.)] and oblate [Aequorea victoria (Murbach and Shearer, 1902), Phialidium gregarium (A. Agassiz, 1862)] species from a guild of co-occurring medusan predators which are seasonally abundant in the waters at Friday Harbor, WA, USA.

In order to evaluate the effect of differences in bell contraction patterns on swimming, we accompanied bell motion measurements with a measure of the energetic efficiency of swimming by calculating the Froude propulsion efficiency $\left(\mathrm{Fr}_{\mathrm{p}}\right)$. A highly efficient propulsive mechanism yields an increase in the velocity of a swimmer equivalent to that of the water moved backwards in the wake by the act of swimming. $\mathrm{Fr}_{\mathrm{p}}$ compares the velocity of an object with that of the objects wake (Vogel, 1994a) and provides an index swimming performance with which to compare medusae of different bell morphologies.

A.

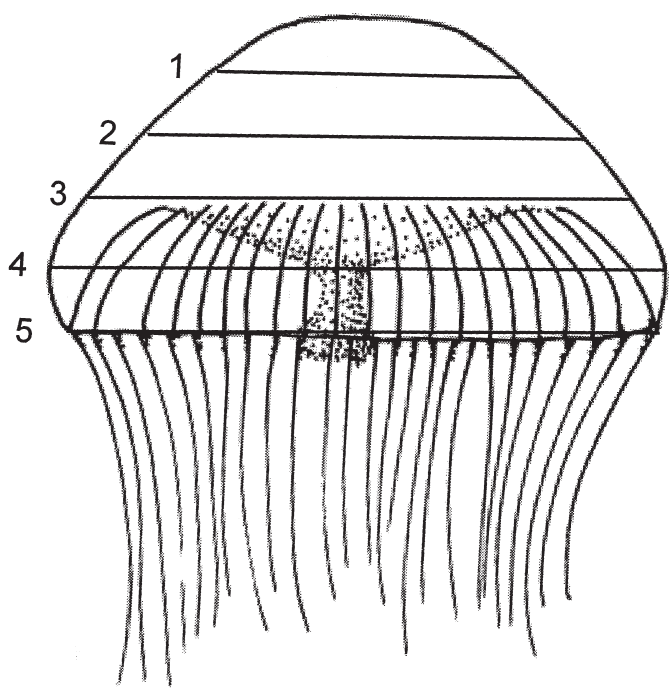

\section{METHODS}

Changes in bell morphology during swimming were measured for three individuals of each hydromedusan species. Swimming by the medusae was recorded using a backlit optical system (Costello and Colin, 1994). Medusae were observed while swimming freely in 0.22 um filtered seawater within rectangular vessels ranging in dimensions from $4.5 \times 8.0$ x $2.0 \mathrm{~cm}$ to $25.5 \times 30.5 \times 18.5 \mathrm{~cm}$ (width $\mathrm{x}$ height $\mathrm{x}$ depth) and volumes from 50-14,000 ml. Vessel choice depended upon medusa diameter. Artemia salina eggs were used as tracers of fluid movements within the vessels. Particle motions were used to determine the velocity of the jet produced by the contracting medusae.

Cross-sectional measurements were made at five different points along the oral-aboral axis of the bell to determine the differences in contraction patterns between the oblate and prolate forms. In order to accommodate the different sizes of the various medusae, five equidistant sampling points were determined for each individual medusa based on the maximum bell height of that medusa (Fig. 1). As a medusa's bell deformed during the pulsation cycle, the locations of the cross-sections maintained their relative spacing with reference to the bell margin. Changes in cross-sectional dimensions of each of the five bell locations were measured throughout one complete bell pulsation cycle for three individuals of each species of hydromedusae. Measurements of cross-sectional widths were made with image analysis software (Optimas Corporation). A

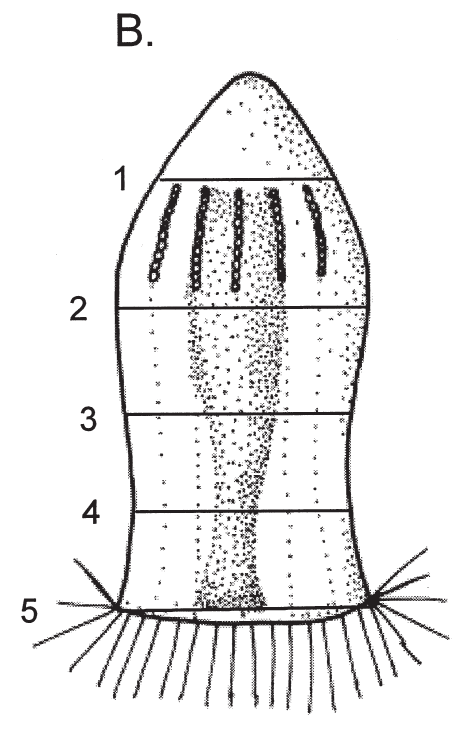

FIG. 1. - Representative cross-sectional sample locations along the bells of (A) Aequorea victoria and (B) Aglantha digitale. 

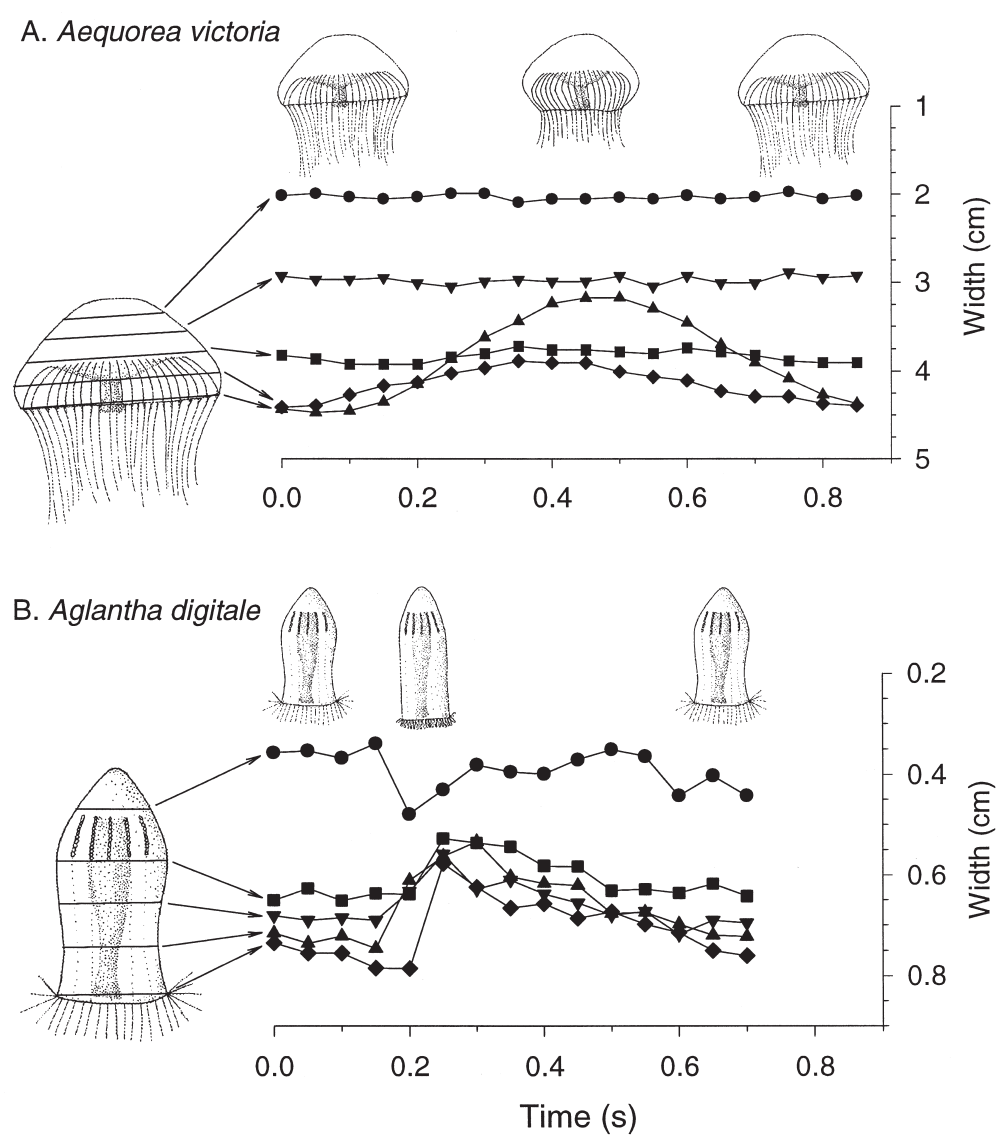

FIG. 2. - Typical cross-sectional widths (cm) during pulsation cycle for the hydromedusae (A) Aequorea victoria and (B) Aglantha digitale. Cross-sectional widths were measured every $0.33 \mathrm{sec}$ during swimming at five evenly spaced locations along the height of the bell. Numbers along the medusa's bell indicate the position above the bell margin $(\mathrm{cm})$ of each cross section.

metric scale was included within videotaped sequences in order to allow spatial calibration of the video sequences. Motion only within the twodimensional viewing field was assured by using a sequence in which bell orientation was level and the medusa swam from bottom to top of the viewing field.

Froude propulsion efficiency $\left(\mathrm{Fr}_{\mathrm{p}}\right.$; Vogel, 1994a) measures the energetic efficiency of thrust production by an organism or vehicle and was employed in order to compare swimming efficiencies of medusae possessing different bell forms. A medusa's velocity was measured as the rate of change in the position of the anterior point of the bell during the contraction phase of the pulsation cycle. The tip of Aglantha's bell was sometimes difficult to locate precisely due to its transparency, so change in position of the bell margin was used to measure medusan velocity for this species. Jet velocity was determined by tracking particles (6-8 for each medusa) ejected through the velar aperture at the beginning of the contraction phase. $\mathrm{Fr}_{\mathrm{p}}$ was calculated by comparing the medusa's body velocity $\left(\mathrm{V}_{\mathrm{m}}, \mathrm{cm}\right.$ $\mathrm{sec}^{-1}$ ) with the velocity of the jet expelled by the medusa $\left(\mathrm{V}_{\mathrm{j}}, \mathrm{cm} \mathrm{sec}^{-1}\right)$, where:

$$
\mathrm{Fr}_{\mathrm{p}}=\frac{2 \mathrm{~V}_{\mathrm{m}}}{\mathrm{V}_{\mathrm{j}}+\mathrm{V}_{\mathrm{m}}} \times 100
$$

$\mathrm{Fr}_{\mathrm{p}}$ was measured for 10-14 individuals of each species of hydromedusae.

Statistical analysis (Statistica, Statsoft Inc.) relied upon ANOVA of $\mathrm{Fr}_{\mathrm{p}}$ and changes in crosssectional dimensions during bell contraction. Differences between cross-sectional samples within a species were tested using Tukey's honest significant difference test (Tukey's HSD) and a value of $a=0.05$ was used as the critical value determining statistical significance.

\section{RESULTS}

Prolate medusae contracted their bells differently during swimming than did oblate medusae. Figure 2 illustrates representative examples of bell shape 
A. Aequorea victoria

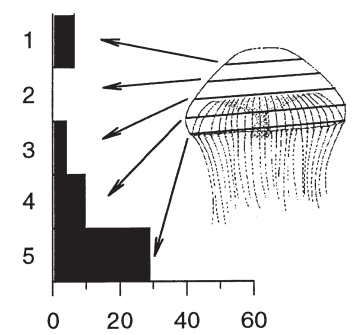

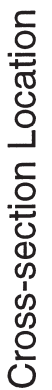
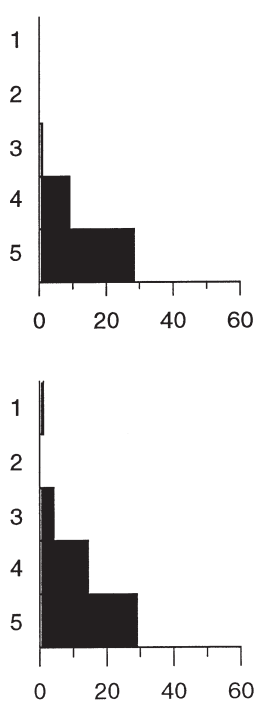

B. Phialidium gregarium
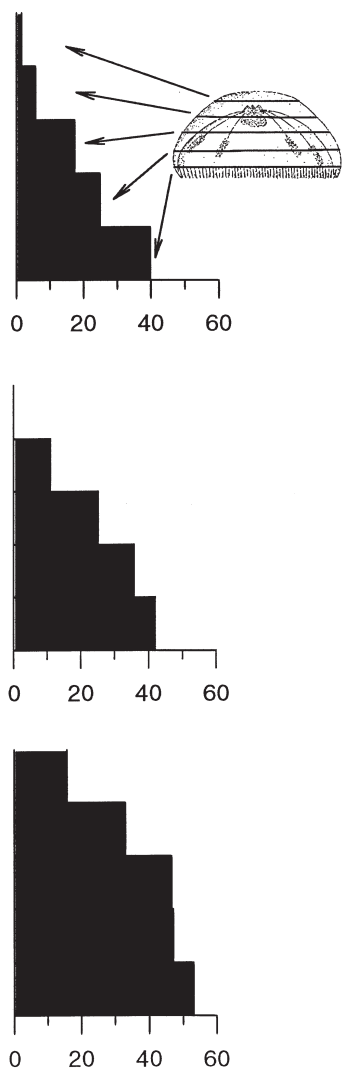

C. Sarsia sp.
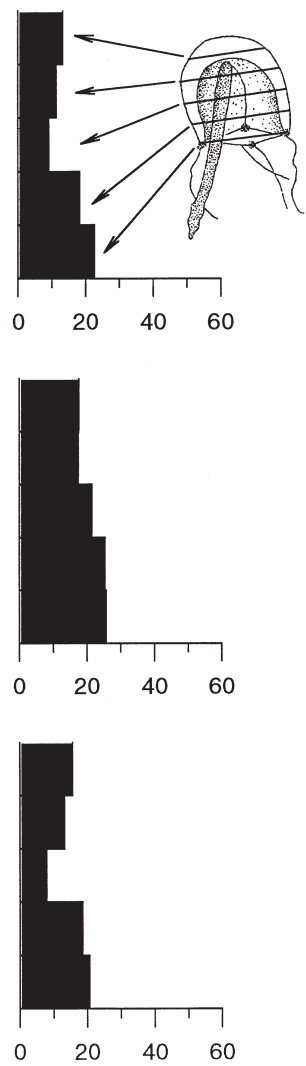

D. Aglantha digitale
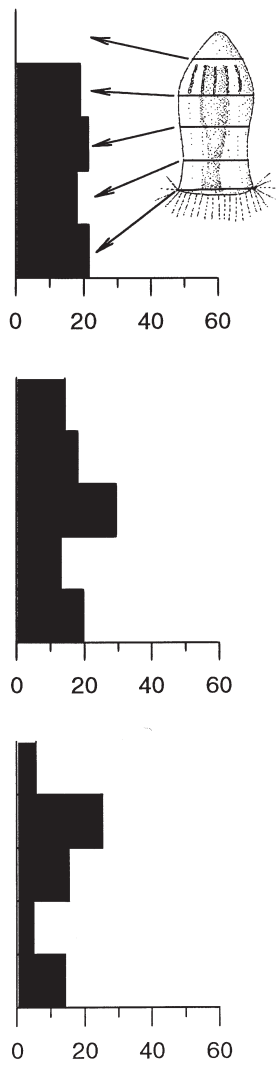

\section{Bell Contraction (\%)}

FIG. 3. - Percent contraction relative to resting bell width at different locations along the bells of (A) Aequorea victoria, (B) Phialidium gregarium, (C) Sarsia sp. and (D) Aglantha digitale. These values represent the total percent change in bell cross section that occurred over the course of bell contraction. Contraction lengths have been scaled to bell diameter for each individual hydromedusa.

alterations during swimming by Aglantha digitale and Aequorea victoria. Cross-sectional dimensions of Aglantha's bell decreased essentially uniformly along the length of the bell during contraction. The anterior tip of the bell was an exception to this pattern because it appeared to bulge slightly as the rest of the bell contracted to its narrowest dimensions. In contrast, cross-sectional dimensions demonstrated that Aequorea's bell contracted almost solely at the bell margin. Bell cross-sectional dimensions varied most nearest the bell margin but there was little or no change at the remaining three sampling locations that were successively more distant from the bell margin. Measurements of these individuals indicated that the jet expelled by Aglantha was created by a contraction that was evenly distributed along the full length of the bell while Aequorea used only the portion of the bell closest to its margin.

Further comparisons within and between medusan species required standardization of dimensional measurements for individuals of different sizes. For this reason, the change in width of each bell crosssection during bell contraction was expressed as a percentage of the relaxed (prior to contraction) bell width for that cross-section. This conversion from absolute lengths to a non-dimensional percentage allowed statistical comparisons of morphological changes by different size individuals of one species as well as between different species with divergent bell morphologies.

Comparisons of standardized cross-sectional data of medusae confirmed that bell contraction patterns differed significantly between genera and location along the bell (ANOVA, $p<0.001$ for both variables). Although contraction patterns showed some intraspecific variation (Fig. 3), the average percent contraction (Fig. 4) was not significantly different for different locations along the bells of Aglantha (ANOVA, $\mathrm{p}=0.088$ ) or Sarsia (ANOVA, $\mathrm{p}=0.066$ ). In contrast, average percent contraction varied significantly along the bells of Phialidium (ANOVA, $\mathrm{p}$ $=0.014$ ) and Aequorea (ANOVA, $\mathrm{p}<0.001$ ). For 


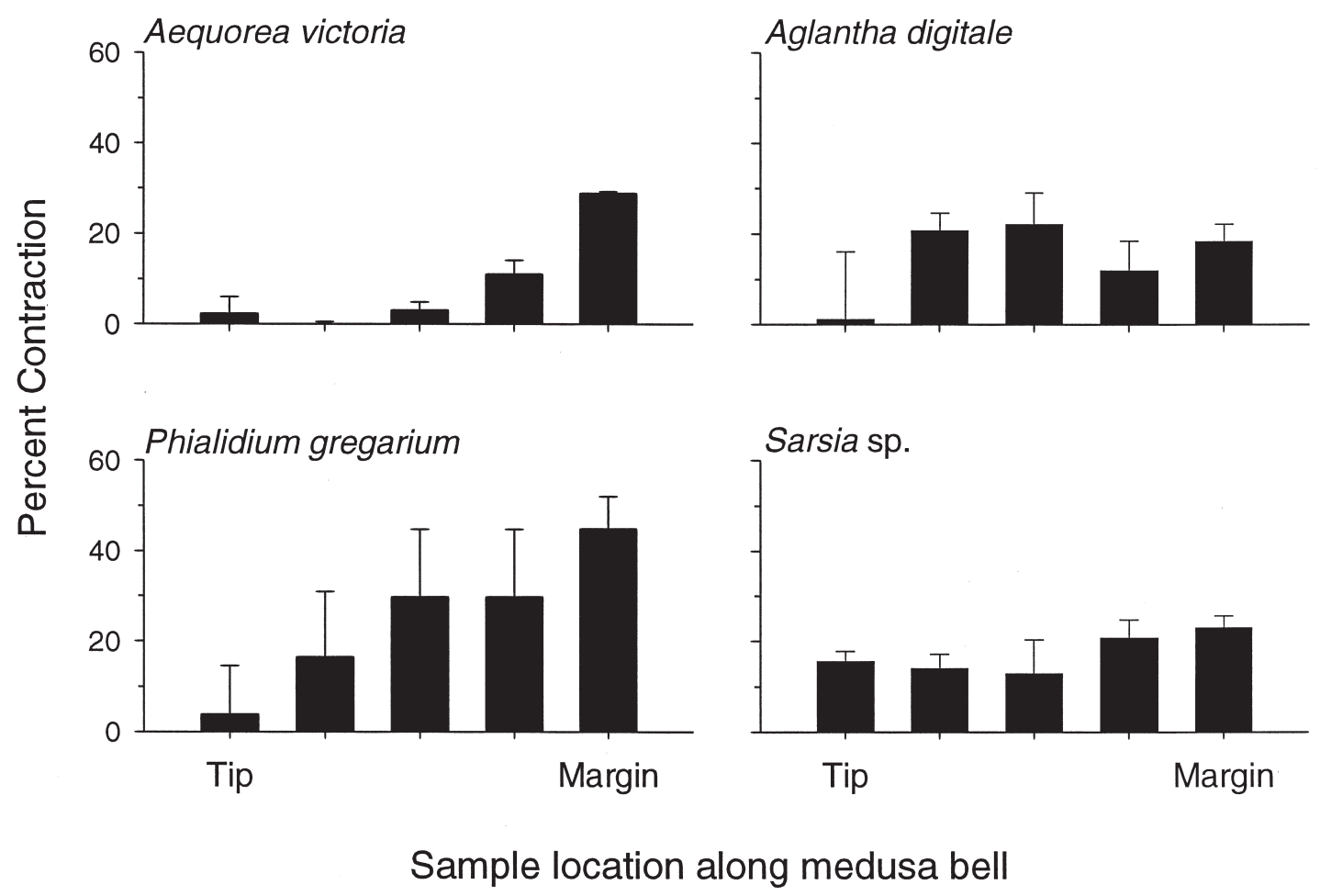

FIG. 4. - Average percent change in cross-sectional width of medusae during bell contraction. Values represent mean $( \pm 1$ std dev) of three individual medusae sampled at each location spanning the bell tip to the bell margin.

both the latter species, contraction was greatest at the bell margin. However, the two oblate species differed significantly (ANOVA, $p=0.002$ ) in the manner of bell contraction. The extent of contraction increased from the distal tip to the bell margin of Phialidium. In contrast, contraction by Aequorea was limited essentially to the lower portion of the bell and there were no significant differences between contraction at the bell tip and the nearest two bell sample locations. The overall bell contraction patterns indicated that contraction by the prolate genera was distributed essentially evenly along the bell length whereas oblate genera contracted primarily at the bell margin. Greater variation occurred within the oblate than the prolate species.

\section{Froude propulsion efficiencies}

The manner in which the medusae accelerate water to generate thrust during swimming, as measured by Froude propulsion efficiency $\left(\mathrm{Fr}_{\mathrm{p}}\right)$, was distinctly different (ANOVA, $\mathrm{p}<0.001$ ) for prolate and oblate medusae. There were no significant differences between the two prolate genera (ANOVA, $\mathrm{p}>0.999$, Fig. 5) or the two oblate genera (ANOVA, $\mathrm{p}>0.999$, Fig. 5). However, the oblate $\left(\mathrm{Fr}_{\mathrm{p}}\right.$ avg. $=$ 97\%) medusae had significantly higher efficiencies

\section{Froude Propulsion Efficiencies}

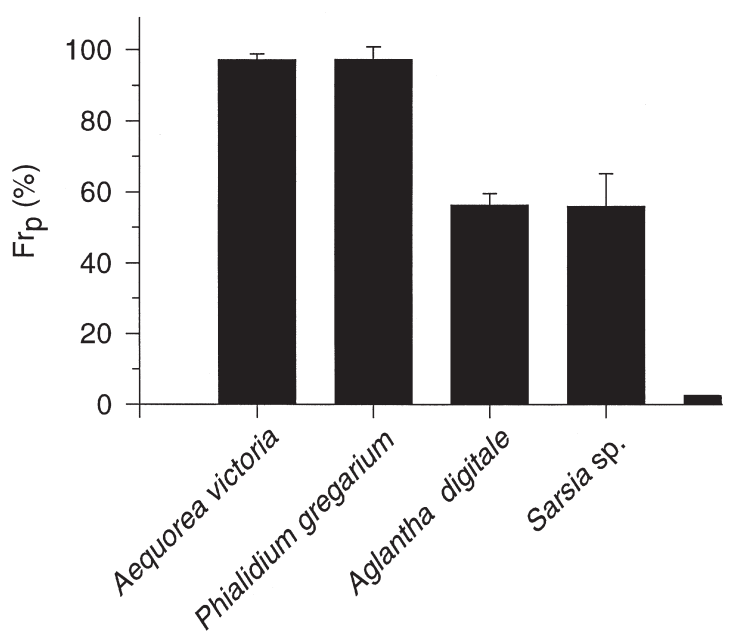

FIG. 5. - Froude propulsion efficiencies ( $\mathrm{Fr}_{\mathrm{p}}$ ) of hydromedusae. Bars represent the mean of between 10-14 individuals of each species, error bars indicate \pm 1 standard deviation about the mean.

(ANOVA, $\mathrm{p}<0.001)$ than did the prolate $\left(\mathrm{Fr}_{\mathrm{p}}\right.$ avg. $=$ $56 \%)$ medusae.

DISCUSSION

Oblate and prolate shaped medusae function differently and these differences are expressed both in the mechanical nature of bell pulsation and in swim- 
ming performance. Prolate medusae such as Aglantha digitale and Sarsia sp. contract the entire bell rapidly and essentially evenly along the full bell length, producing a high velocity, narrow stream of water jetting through the velar aperture and thrusting the medusa forward. The resulting accelerations of the medusa are relatively rapid (Daniel, 1983; Costello and Colin, 1996). However, from an energetic perspective, the process is inefficient by comparison to that of the oblate genera. The high velocity jet is energetically costly because the energy expended to accelerate a fluid increases as the square of the increase in velocity (Vogel, 1994a). Therefore, each incremental increase in the velocity of the jet costs an exponential increase in energy expenditure. The high cost per yield in forward motion of the medusa is expressed by the Froude propulsion efficiency, which is significantly lower for the prolate than for the oblate medusae. However, the low efficiency of jet propulsion of prolate medusae may be justified, in an evolutionary sense, by the advantages gained by rapid acceleration during escape from potential predators. Further, because they are ambush predators, swimming comprises a relatively low proportion of the time budget of these medusae (Costello and Klos, unpublished data) and, therefore, may represent a low expense in their composite energy budget.

The oblate medusae provide an informative contrast with the prolate forms. Bell contraction of Aequorea victoria and Phialidium gregarium occurs primarily near the bell margin. For Aequorea, the remainder of the bell moves little during contraction. The resulting stream of water ejected through the wide velar aperture is relatively wide and slow moving. The low velocities of the wake require less energy during acceleration of the fluid than high velocity wakes of prolate medusae. The wake velocities of the oblate medusae are similar in magnitude to the forward velocities of the medusan bells. The lower energy expenditure required for acceleration to these low velocities is expressed in the high Froude propulsion efficiencies of the oblate medusae. Essentially, the oblate medusae accelerate a much larger relative volume of water to a slower velocity than do the prolate forms. The lower acceleration minimizes the energy necessary to move a given volume of fluid; the larger fluid volume accelerated ensures adequate thrust to move the medusan body forward. The high efficiency and low energetic cost of this form of propulsion suits a foraging mode dependent upon continuous swimming. Further, because prey capture depends upon entrainment in the flow created during swimming, movement of larger volumes of fluid during bell pulsation contributes to the success of the cruising foraging strategy evolved by these oblate medusae. The tradeoff inherent in this swimming pattern is the loss of rapid acceleration for escape swimming. Apparently, this has not been the dominant selective force shaping evolution of oblate hydromedusae.

Rather than being simply isolated traits of variably shaped medusae, we suggest that the differences in bell contraction and propulsive efficiencies reflect the evolution of different propulsive systems by oblate and prolate medusae. Prolate medusae use their entire bell to contract a clearly bounded fluidfilled chamber and expel that fluid through a narrow orifice. This propulsive system is a classical example of the jet propulsion most commonly associated with medusae (Daniel, 1983; Denny, 1993; Vogel, 1994a, 1994b). Such propulsive systems are typified by low Froude propulsive efficiencies (Vogel, 1994a). By comparison, the subumbrellar cavities of oblate hydromedusae are less clearly bounded by their wide velar apertures (Costello and Colin, unpublished data). The relative importance of jet production for propulsion of these medusae has not been definitively measured. However, flapping of the bell margin during contraction clearly contributes significantly to wake production by large oblate medusae. Flows created by entraining fluid adjacent to the bell margin carry prey items into the tentacle mass trailing the swimming medusae. Propulsion by flapping a broad, flattened bell in this manner is more consistent with a drag-based propulsive system which creates thrust by pushing against a fluid (Denny, 1993). The resistance of the fluid to shear by the flapping body generates an opposite reactive force, thrust, which moves the medusa forward.

The advantages of this propulsive mode for oblate medusae are twofold. First, the flow generated during bell contraction entrains and transports prey to the medusa's tentacles. Swimming thus becomes an integral part of foraging. Second, oblate morphologies can result in substantial hydrodynamic advantages compared to prolate forms for medusae which are cruising predators. The most extreme examples of this coupling of oblate morphology with a cruising foraging mode occur in the scyphomedusae. Pelagic representatives of this cnidarian class have no velum, and thus no orifice for jet propulsion, and depend completely on flows produced during essentially continuous swimming 
(Costello et al., 1998) to carry prey to capture surfaces (Costello and Colin, 1994, 1995).

Whereas all pelagic scyphozoan classes exhibit drag-based propulsion coupled with a cruising foraging mode, the proportion of hydromedusae utilizing this method of propulsion appears to be low. Most adult hydromedusae are relatively small $(<3.0 \mathrm{~cm}$ bell diameter) and large, oblate forms appear to have arisen primarily within the Leptomedusae (Costello and Colin, unpublished, based on Mayer, 1910). Thus, as a group, the hydromedusae are probably dominated by jet propulsion and the drag-based propulsion of large, oblate genera is less common. The ecological consequences of these morphological and functional patterns are significant and affect patterns of prey selection (Mills pers comm.; Costello and Colin, unpublished data). These interrelationships will need to be elaborated in order to fully understand the role played by hydromedusae in their natural environment.

\section{ACKNOWLEDGEMENTS}

The authors thank two anonymous reviews for their instructive comments. This research was supported by grants OCE-9103309 and OCE-9820172 from the US National Science Foundation to JHC.

\section{REFERENCES}

Colin, S.P. and J.H. Costello. - 1996. Relationship between morphology and hydrodynamics during swimming by the hydromedusae Aequorea victoria and Aglantha digitale. Sci. Mar. 60: 35-42.

Costello, J.H. - 1992. Foraging mode and energetics of hydrozoan medusae. Sci. Mar. 56: 185-191

Costello, J.H. and S.P. Colin. - 1994. Morphology, fluid motion and predation by the scyphomedusa Aurelia aurita. Mar. Biol. 121:327-334

Costello, J.H. and S.P. Colin. - 1995. Flow and feeding by swimming scyphomedusae. Mar. Biol. 124:399-406

Costello, J.H., E. Klos and M.D. Ford. - 1998. In situ time budgets of the scyphomedusae Aurelia aurita, Cyanea sp., and Chrysaora quinquecirrha. J. Plankton Res. 20: 383-391.

Daniel, T.L. - 1983. Mechanics and energetics of medusan jet propulsion. Can. J. Zool. 61: 1406-1420.

Daniel, T.L. - 1984. Unsteady aspects of aquatic locomotion. Amer. Zool. 24: 121-134.

Daniel, T.L. - 1985. Cost of locomotion: unsteady medusan swimming. J. Exp. Biol. 119: 149-164.

Denny, M.W. - 1993. Air and water. Princeton Univ. Press, Princeton, USA

Kramp, P.L. - 1959. The Hydromedusae of the Atlantic Ocean and adjacent waters. Dana-Rep., 46: 1-283.

Mayer, A.G. - 1910. Medusae of the World. Carnegie Inst. of Washington, Washington, D.C.

Russell, F.S. - 1953. The medusae of the British Isles. Anthomedusae, Leptomedusae, Limnomedusae, Trachymedusae and Narcomedusae. Cambridge University Press, London.

Vogel, S. - 1994a. Second-rate squirts. Discover 15: 72-76.

Vogel, S. - 1994b. Life In Moving Fluids: The physical biology of flow. Princeton Univ. Press, Princeton, USA. 467 pp. 